\title{
Betreuung, Erziehung, Bildung
}

\section{Ganzheitliches Präventionsnetz zur Sicherung gelingender kindlicher Entwicklungsverläufe in den ersten zehn Lebensjahren}

\author{
Susanne Gastmann
}

Dr. Susanne Gastmann ist Diplom-

Ökotrophologin mit dem Schwerpunkt Haushaltswissenschaften. Derzeit ist sie Koordinatorin des Projektes »Hallo Welt - Familien begleiten « für die Stadt und den Landkreis Gießen.

E-Mail Susanne.Gastmann@

haushalt.uni-giessen.de
Familie ist zu einer zeitintensiven komplexen Herstellungsleistung geworden, so dass passgenaue familienunterstützende und familienbegleitende Angebote in ihrem Umfeld einen erheblichen Bedeutungszuwachs erfahren.

Ein Gelingen kindlicher Entwicklungsverläufe ist für die Familien eine Lebensaufgabe und für die Gesellschaft ein besonderes Anliegen. Der tiefgreifende Strukturwandel von Kindheit und Familie als Folge des Übergangs von der Industriegesellschaft zur wissensbasierten Dienstleistungsgesellschaft hat dabei jedoch vor allem zu gestiegenen Anforderungen an die Familienmitglieder geführt. Sie treffen heute auf vielfältige Schwierigkeiten, ihren Alltag verlässlich zu koordinieren und ihre sozialen Beziehungsnetze zu pflegen.

Betreuung, Erziehung und Bildung gehören im Verlauf der kindlichen Entwicklung innerhalb der Familien zu den Grundlagen im alltäglichen Zusammenleben. Stichworte wie beispielsweise niedrige Geburtenrate, Vernachlässigung und Misshandlung von Kindern, überforderte Eltern und gravierende Verhaltensauffälligkeiten der Kinder in Kindergarten und Grundschule lassen erkennen, dass diese Grundlagen neu überdacht werden müssen.

Der Gestaltungsspielraum der familiären Verantwortung ist, neben den familialen Alltagsbedingungen, wie den verfügbaren Ressourcen, den Lebenseinstellungen der Eltern sowie deren Handlungsspielräumen, eng verbunden mit den gesellschaftlichen Rahmenbedingungen und Unterstützungsangeboten. Merkmal der vielen Angebote ist jedoch, eine insgesamt einseitige Problemwahrnehmung entweder der Betreuungsaspekte oder der Erziehungsaspekte oder der Bildungsaspekte. Trotz der zentralen Bedeutung, die die Familien und die Eltern für die Entwicklungschancen der Kinder haben, ist ihre feste Einbindung in das soziale Netzwerk noch nicht vollständig realisiert.
In Zeiten, in denen vor allem die internationale Konkurrenz immer größer wird, ist eine qualitativ hochwertige Bildung von Anfang an ein entscheidender Standortvorteil für Deutschland und sollte als Produktivkraft Nummer eins angesehen werden. Der Ausbau der Wissensgesellschaft ist das oberste Ziel der erneuerten Lissabon-Strategie der Europäischen Union, und auch Deutschland hat sich diesem Ziel in seinem nationalen Reformprogramm der Bundesregierung erklärt. (Statistisches Bundesamt 2006:33-37).

Doch welche Voraussetzungen, gestützt auf einem haushaltswissenschaftlichen Bezugsrahmen, müssen gegeben sein, damit kindliche Entwicklungsverläufe in den ersten zehn Lebensjahren im sozialen Nahraum besser gelingen können? Hier zeigen die Ergebnisse meiner Dissertation deutlich, dass die Familien und das Familienhaushaltssystem als Ort gelingender kindlicher Entwicklung im Mittelpunkt der Betrachtungsweise stehen sollten. Eine nur kindzentrierte oder nur elternzentrierte oder nur einrichtungsorientierte Sicht greift wesentlich zu kurz.

\section{Herkunftsfamilie als wichtigste Sozialisationsinstanz}

Zahlreiche Wissenschaften setzen sich mit der Familie in all ihren Erscheinungsformen auseinander und akzentuieren unterschiedlich. Um eine Einseitigkeit fächerspezifischer Diskussionen zu begegnen und die Komplexität zu verdeutlichen, ist in der folgenden Definition von Familie ein breites Spektrum nebeneinander bestehender Ansichten eingeflossen:

Die Familie stellt im Entwicklungsverlauf der Kinder den entscheidenden Bezugspunkt dar. Sie besteht aus mindestens zwei Generationen, deren enge, fürsorgliche und emotional gewachsene Beziehung zwar in der Form des Zusammenlebens variiert, aber immer einen gemeinsamen Lebens- und Alltagsbezug hat. Die 


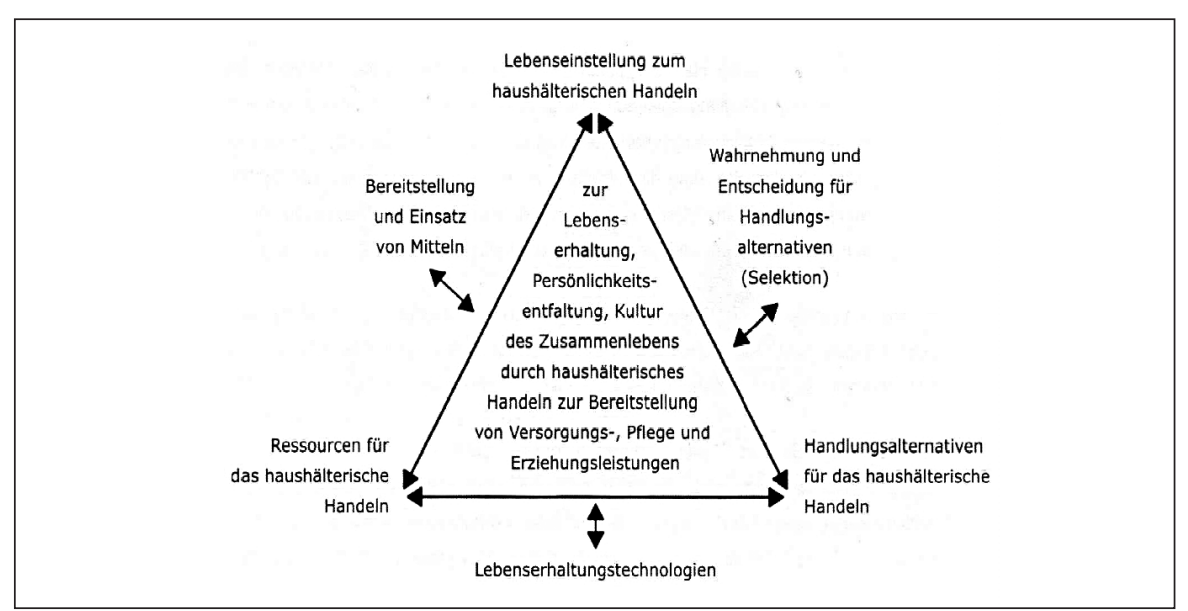

Abb. 1: Im »Haushälterischen Dreieck « lassen sich die Aufgaben im Familienhaushalt darstellen.

Quelle: v. Scheitzer 1991:138
Familie muss im Kontext eines sozialen Netzes, beispielsweise mit den Großeltern, gedacht werden und ist darüber hinaus in gesellschaftliche Rahmenbedingungen eingebettet und wird von diesen unterstützt. Das Fundament zur Erbringung von fürsorglicher Beziehungsarbeit, die Rückhalt, Schutz und Sicherheit im Prozess des Aufwachsens schenkt, bildet das haushälterische Handeln aller beteiligten Familienmitglieder, welches durch typische Zeitverwendungsmuster für die familiale Gruppe bestimmt und personell nur begrenzt austauschbar ist. Die Familie ist also ein Ort, an dem Generationen füreinander Verantwortung übernehmen, und kann somit als »ein soziales Netzwerk besonderer Art « (Meier-Gräwe 2009a:10) aufgefasst werden.

Für ein Gelingen kindlicher Entwicklungsverläufe stehen die Herkunftsfamilien mit ihren jeweiligen Alltagskompetenzen, Lebens- und Bedarfslagen im Mittelpunkt. Familienhaushalte sind darauf aufbauend eine Form von privaten Haushalten, in denen mindestens eine Elternteil-Kind-Gemeinschaft, also eine Fa-

milie, zusammenlebt (Mikrozensus 2006:16). Familiale Leistungen kommen dabei den Familienmitgliedern selbst zugute und zeigen positive wie negative Effekte für Gesellschaft, Staat und Wirtschaft (Graßl 2000). So lassen sich haushälterische Handlungen nach ArbeitsFunktions- und/oder Dispositionsbereichen zur Bereitstellung von Versorgungs-, Pflege- und Erziehungsleistungen für den Familienhaushalt im »Haushälterischen Dreieck « darstellen (vgl. Abb. 1).

Diese Handlungen sind an die elterlichen Ressourcen, Lebenseinstellungen und Handlungsalternativen gebunden und wirken sich ganz unmittelbar auf die Lebensqualität, Lebensführung und den gesamten Lebensverlauf ab der Geburt des Kindes aus. Neben den finanziellen Ressourcen sind es auch beispielsweise die Bildungsressourcen der Eltern, die eine entscheidende Bedeutung für die kindliche Entwicklung haben.

Am Beispiel von in Armut lebenden Familien wird Folgendes deutlich: Nach den Angaben des Statistischen Bundesamtes leben aktuell 2,5 Millionen Kinder in

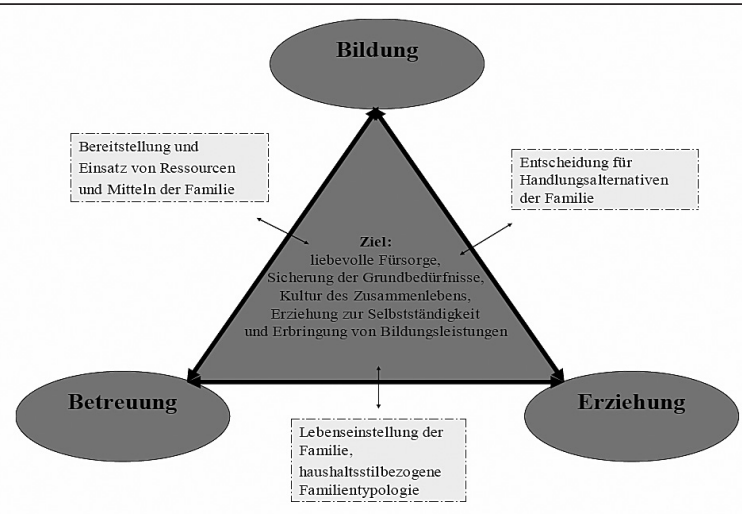

Abb. 2: Das »Familiale Dreieck « zeigt die Zusammenhänge bei der Erfüllung des Betreuungs-, Erziehungs- und Bildungsauftrags von Familien.
Deutschland auf Sozialhilfeniveau oder knapp darüber. Das ist jedes sechste Kind in Deutschland (Statistisches Bundesamt 2009). Aus Armutsstudien wissen wir, dass oft weniger die finanzielle Lage einer Familie die Lebenschancen negativ beeinflusst, als vielmehr die fehlende Weitergabe von Humanvermögen in Form von allgemeinen Lebenskompetenzen (Meier-Gräwe 2006: 7). So kann davon ausgegangen werden, dass arme Kinder bezüglich Betreuung, Erziehung und Bildung von Anfang an benachteiligt sind und dass Armut ein großes Risiko für gelingende kindliche Entwicklung ist, wobei jede Familie von Armut betroffen werden kann, denn »Armut hat viele Gesichter « (Meier-Gräwe 2009b:32).

Betreuung, Erziehung und Bildung stellen im Lebensverlauf der Familie ganz unterschiedlich intensive Anforderungen an die Familienmitglieder und sind nicht voneinander zu trennen, sie bedingen sich also gegenseitig und bilden eine Einheit. Das »Familiale Dreieck« (vgl. Abb. 2) unterstreicht die Zusammenhänge bei der Erfüllung des Betreuungs-, Erziehungsund Bildungsauftrags der Familien und zeigt auf, bei welchen Problemlagen aufgrund mangelnder haushälterischer Handlungen Unterstützung erfolgen muss. Ähnlich wie beim »Haushälterischen Dreieck « durch von Schweitzer liegt in jeder haushälterischen Handlung für Betreuung, Erziehung und Bildung eine tiefere Bedeutung. Jede Handlung in diesem Sinne bedarf im Familienhaushalt eines Einsatzes von Ressourcen und Mitteln und besitzt Alternativen. Trotzdem besteht bei haushälterischen Handlungen für Betreuung, Erziehung und Bildung eine Einheit, indem das Kind also betreut wird, wird es erzogen und gebildet. Wenn das Kind erzogen wird, wird es betreut und gebildet, und wenn das Kind gebildet wird, wird es erzogen und betreut. Wenn also bei diesen Beziehungen im Laufe der kindlichen Entwicklung eine Handlung im Vordergrund steht, werden trotzdem auch die beiden anderen mit einbezogen. Aus diesem Grunde ist es für den kindlichen Entwicklungsverlauf wichtig, dass sich alle beteiligten Akteure darüber im Klaren sind, dass sie die Schwerpunkte so setzen, dass dabei die Einheit von Betreuung, Erziehung und Bildung gewährleistet ist. Aus diesen Zusammenhängen ist ein fundiertes Wissen über das haushälterische Handeln unabdingbar für die gelingende Gestaltung des Lebensverlaufes. 


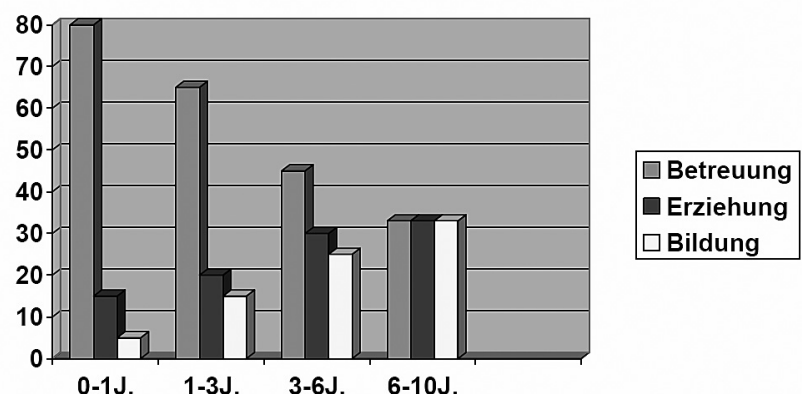

Abb. 3: Die Gewichtung der Betreuungs-, Erziehungs- und Bildungsaufgaben in der Familie ändert sich mit dem Lebensalter des Kindes.

Wie lassen sich jedoch in dieser Diskussion die Begriffe Betreuung, Erziehung und Bildung voneinander abgrenzen? Folgende Kurzzusammenfassung zeigt Schwerpunkte auf: Gelingende Betreuung zeichnet sich vor allem durch liebevolle Fürsorge und die Erfüllung der Grundund Sicherheitsbedürfnisse aus, das heißt zum Beispiel Pflege, Schutz, Nahrung, Liebe, Schlaf, Achtung und soziale Anerkennung mit dem Ziel, eine vertrauensvolle Beziehung zum Kind aufzubauen. Erziehung gelingt, wenn der Heranwachsende in seiner charakterlichen Entwicklung unterstützt, gefördert und einfühlsam begleitet wird mit dem Ziel, soziales Verhalten zu erlernen sowie selbstständig und eigenverantwortlich zu handeln. Dafür gilt es Normen, Werte, Regeln vertraut werden zu lassen. Gelingende Bildung hingegen beschreibt die lebenslange Entwicklung eines Menschen im Hinblick auf die Entfaltung seiner geistigen, seelischen, kulturellen und sozialen Fähigkeiten. Dies erfolgt zum einen über die Entfaltung und Schulung der inneren Kräfte zur Entwicklung der Persönlichkeit und

zum anderen durch das Kennenlernen und Erschließen der äußeren Welt wie Sprache, Wissen und Kultur. Bildung ist demnach nicht allein die Wissensvermittlung durch Dritte.

Der Betreuungs-, Erziehungs- und Bildungsaufwand für Kinder besitzt im Lebensverlauf der Familie unterschiedliche Prioritäten. Aus den Untersuchungen, anhand von Fragebögen für Eltern, ging hervor, welche Schwerpunkte diese in ihrer Arbeit bei der Betreuung, Erziehung und Bildung im Laufe der kindlichen Entwicklung setzten (vgl. Abb. 3).

Obgleich alle drei Schwerpunkte - Betreuung, Erziehung, Bildung - von Anfang an eine bedeutende Rolle spielen, lässt ihre Gewichtung innerhalb der familialen Arbeit erkennen, dass nach der Geburt des Kindes bis zum Ende der Kindergartenzeit der Betreuungsanteil bei den Tätigkeiten der Eltern für die Kinder am höchsten ist. Der Erziehungs- als auch der Bildungsanteil der elterlichen Arbeit nimmt im Laufe der Entwicklung des Kindes jedoch kontinuierlich zu. Im Grundschulalter ist der Betreuungs-, Er-

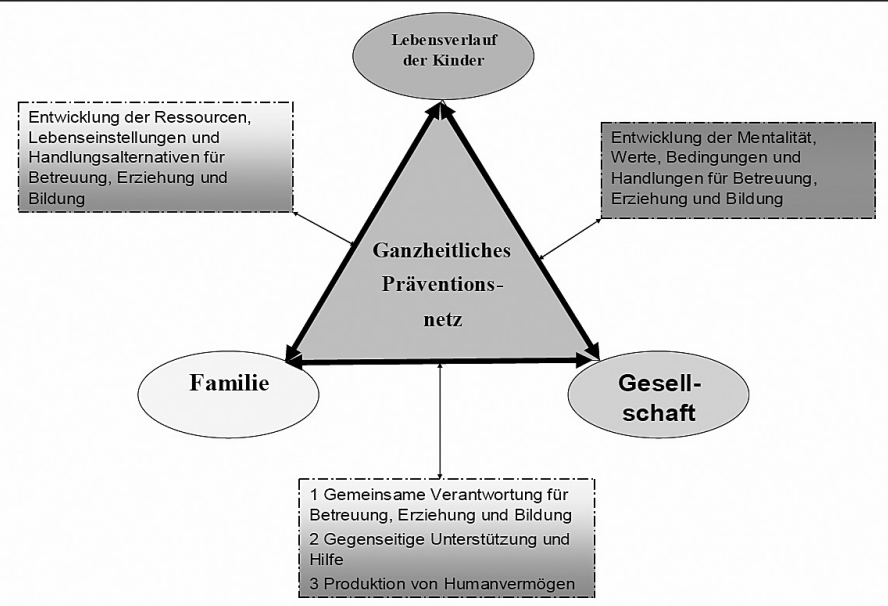

Abb. 4: Kommunikationsprozesse im Familienhaushalt bestimmen die Entscheidungen zu Fragen von Betreuung, Erziehung und Bildung der Kinder. ziehungs- und Bildungsanteil bei den Tätigkeiten der Eltern für das Kind in etwa gleich.

Je früher ein gesellschaftlicher Akteur eine Familie bei ihren Aufgaben unterstützt, umso kompatibler sollte sein Angebot mit den Lebenseinstellungen der Familie sein. Die Eltern gilt es, bei der Angebotssuche niedrigschwellig, vertrauensvoll und passgenau zu unterstützen. Die Aufgabe der Kindergärten ist es, den Eltern bei der Erziehung ihrer Kinder zu helfen sowie ihre Resilienz zu stärken. Die Grundlage für die Erziehung ist eine gute Betreuung. Insofern hat der Kindergarten auch eine sehr wichtige Betreuungsaufgabe. Da der Bildungserfolg der Kinder in dieser Zeit für die Eltern an Bedeutung gewinnt, muss der Kindergarten ebenfalls Bildung vermitteln, vor allem in Vorbereitung auf die Schule. Aufgabe der Schule ist es, die Bildung der Kinder sicherzustellen, wozu gute Erziehung und Betreuung Voraussetzung sind. Im Zuge der Entwicklung zur Ganztagsschule muss auf diese Voraussetzungen geachtet und das geeignete Personal gefunden werden, da die Lehrerinnen den Bildungsauftrag zu erfüllen haben, und dieser leidet, wenn sie verstärkt Erziehungsarbeit leisten müssen.

Durch Betreuungs-, Erziehungs- und Bildungspartnerschaften aller Akteure in der Gesellschaft wird die Chancengleichheit aller Kinder gewährleistet. Die Wahlfreiheit der Eltern über die gesellschaftlichen Angebote ist dabei Voraussetzung.

Familienunterstützende Maßnahmen sind ebenso vielschichtig zu betrachten wie die Lebenslagen der Familien selbst. Die unterschiedlichen Bedarfslagen der Familien bestimmen die Angebote. Es gilt, benachteiligte Familien nicht zu stigmatisieren, sondern eine Balance zwischen Verpflichtung und Selbstbestimmtheit für die Familien zu finden. Die sozialen Dienste sind daher zukünftig aufgefordert als Verbund ihre $\mathrm{Zu}$ sammenarbeit abzustimmen, um sich so auf die konkreten Bedarfslagen der Familien einstellen zu können. Dieser Verbund ist ein ganzheitliches Präventionsnetzwerk mit folgender Zielstellung: Die Chancengleichheit der Kinder ist nur durch einen Dreiklang von Betreuungs-, Erziehungs- und Bildungspartnerschaften aller familialer und gesellschaftlicher Akteure gewährleistet mit dem Ziel, Familien in ihrer Verantwortung und ihrem 


\section{»Was Familien brauchen «}

1 Die Familie als Ort der liebevollen Fürsorge, der Erziehung zur Selbststän1 digkeit und der Entwicklung der geistigen Fähigkeiten bedarf noch größerer Wertschätzung und Unterstützung durch die Gesellschaft und die Politik, denn die Familie und der Familienhaushalt ist der Mittelpunkt für gelingende kindliche Entwicklungsverläufe.

2 Bei der kindlichen Entwicklung bilden Betreuung, Erziehung und Bildung - eine Einheit. So kann Bildung nur gelingen, wenn ein Kind zufrieden stellend betreut und erzogen ist. Werden die Kinder nicht liebevoll betreut, wird es schwer, ihnen eine gute Erziehung angedeihen zu lassen und der Bildungsprozess wird für die Kinder wesentlich schwieriger. Ein fundiertes Wissen über das haushälterische Handeln ist dabei die Grundlage.

3 Eine enge Kooperation zwischen Kindergarten und Grundschule sowie sozi3. alen Diensten, Vereinen und den Kirchen auf der Grundlage der Einheit von Betreuung, Erziehung und Bildung kann die Lebensbedingungen von Familienhaushalten verbessern.

4. Soziale Dienste entwickeln sich zu individuellen lebenslaufrelevanten Beglei- tern der Familien und verfolgen von Anfang an eine stärkenorientierte und präventive Herangehensweise an ihre Arbeit.

Susanne Gastmann

Empowerment zu stärken. Soziale Dienste entwickeln sich somit zu individuellen lebenslaufrelevanten Begleitern der Familien und verfolgen von Anfang an eine stärkenorientierte und präventive Herangehensweise an ihre Arbeit.

Das Präventionsdreieck (vgl. Abb. 4) verdeutlicht die strategische Ergänzung von Familie und Gesellschaft bei der gelingenden kindlichen Entwicklung. Hierbei wird deutlich, dass aus der Rückkopplung von präventiven Maßnahmen im Zusammenspiel von Familien und Gesellschaft positive Kettenreaktionen für alle Beteiligten resultieren, die sich günstig auf den Lebensverlauf der Kinder und damit der zukünftigen Gesellschaft auswirken.

Erkennbar ist, dass die Entscheidungsfindung zu den Fragen der Betreuung, Erziehung und Bildung der Kinder durch Kommunikationsprozesse im Familienhaushalt bestimmt wird. Diese ist abhängig von den Lebenseinstellungen, Ressourcen und Handlungsalternativen der Familienmitglieder. Wichtig sind aber auch die Mentalität, die Werte sowie die Bedingungen und Handlungen zur Betreuung, Erziehung und Bildung der Kinder durch die Gesellschaft. Beides bestimmt ganz entscheidend den Lebensverlauf des Kindes, seiner Familie und damit letztlich die Entwicklung der Gesellschaft, da die Familie das Humanvermögen der Gesellschaft produziert, ohne dafür immer die entsprechende Anerkennung zu erfahren.
Zur Entscheidungsfindung der Familien und der Gesellschaft hinsichtlich der gelingenden kindlichen Entwicklung bedarf es der Offenheit aller Akteure gegenüber der unbewussten Rückkopplung des Kindes über seinen jeweiligen Entwikklungsstand. Ein Kind wird in eine Familie hineingeboren, es kann sie sich nicht aussuchen - diese Tatsache ist nicht beeinflussbar. Die Verknüpfung von Familie, Kind und Gesellschaft und die damit verbundenen Abhängigkeiten machen deutlich, wie wichtig es ist, eine gemeinsame Grundlage für Betreuungs-, Erziehungs- und Bildungsprozesse der nachwachsenden Generation zu entwickeln, an der sich alle beteiligten familiären und gesellschaftlichen Akteure orientieren können. Eine partnerschaftliche Kommunikation mit den Familien und nicht über die Familien ist ein entscheidender Schritt in diese Richtung.

Damit dieser Schritt gut vorbereitet funktionieren kann, könnte eine zentrale Koordinierungsstelle dem Familienhaushalt und den gesellschaftlichen Akteuren zur Verfügung stehen. Diese müsste bei den Familien spätestens ab der Geburt eines Kindes präsent sein, mit einem Angebotsmix aus standortgebundener und aufsuchender Hilfe, die berät und vermittelt. Dazu ist vor allem das Vertrauen der Familien in diese Koordinierungsstelle wichtig. So wäre es wünschenswert, dass sie überparteilich und überkonfessionell ist. Es erscheint daher sinnvoll, die
Koordinierungsstelle im kommunalen Gesamtkonzept zu etablieren und sie dem Oberbürgermeister direkt zu unterstellen.

Die Familie wird durch die Arbeit der Koordinierungsstelle unter der Leitung einer Familienbeauftragten gestärkt, ganz gleich, welcher sozialen oder ethnischen Herkunft sie ist. Die Unterstützung der Familien in Form von Betreuungs-, Erziehungs- und Bildungsdienstleistungen stärkt die Kinder, die Kommune, das Land, den Bund und letztlich die gesamte Gesellschaft.

\section{Literatur}

Graßl, H (2000): Strukturwandel der Arbeitsteilung - Globalisierung - Tertiarisierung und Feminisierung der Wohlfahrtsproduktion. UVK Verlagsgesellschaft. Konstanz.

Meier-Gräwe, U. (2009a): Was ist Familie? Warum es einer begrifflichen Neujustierung bedarf. In: Archiv für Wissenschaft und Praxis der sozialen Arbeit, Familienpolitik auf dem Prüfstand. 39. Jahrgang. Nr. 2/2009. Druck Verlag Kettler. Berlin.

Meier-Gräwe, U. (2009b): Armutsprävention im Sozialraum - ein Schlüssel zur Verringerung von Bildungsarmut. In: Gesellschaft für sozialen Fortschritt (Hg.): Sozialer Fortschritt. Unabhängige Zeitschrift für Sozialpolitik. Jg.58. Heft 2-3. Februar/März 2009.

Meier-Gräwe, U. (2006): Auf den Anfang kommt es an, Bildungsgerechtigkeit für alle Kinder als zukunftsweisendes Aufgabenfeld einer vorsorgenden Gesellschaftspolitik. In: Hessenreport Nr.39: Bildung - die Herausforderung im 21.Jahrhundert. Presse und Bildung $\mathrm{GmbH}$. Wiesbaden.

Mikrozensus (2006): Bevölkerung und Erwerbstätigkeit - Haushalt und Familien. Ergebnisse des Mikrozensus 2006. Statistisches Bundesamt (Hg.). Wiesbaden.

Schweitzer, R. V. (1991): Einführung in die Wirtschaftslehre des privaten Haushalts: Willhelm Fink Verlag. München. Statistisches Bundesamt (2009): Kinderarmut. [http://www.kinderpolitik.de/ zahlen_daten_fakten/kinderarmut.php, 17.07.2009]

Statistisches Bundesamt (2006)(Hg.): Im Blickpunkt. Deutschland in der EU 2006. Wiesbaden. 